\title{
PLATAFORMA DE AVALIAÇÃO DE FUNÇÕES EXECUTIVAS COM TESTES NEUROPSICOLÓGICOS COMPUTADORIZADOS
}

\author{
Ana Garcia ${ }^{1 *}$, Fabíola Fleury ${ }^{2}$, Gabriel Silva ${ }^{2}$, Hugo Honda ${ }^{3}$ e Maria Clotilde H. Tavares ${ }^{1}$ \\ ${ }^{1}$ Laboratório de Neurociências e Comportamento, Departamento de Ciências Fisiológicas, Instituto de Ciências \\ Biológicas \\ ${ }^{2}$ Engenharia de Software, Faculdade do Gama \\ ${ }^{3}$ Engenharia de Computação, Faculdade de Tecnologia \\ Universidade de Brasília, Brasília, Distrito Federal, Brasil
}

\begin{abstract}
RESUMO
Os testes neuropsicológicos são tarefas elaboradas especificamente para investigação de processos cognitivos relacionados a uma estrutura cerebral específica. Esses testes podem ser utilizados em ensaios clínicos e também para obter resultados em pesquisas neurocientíficas. Especialmente naqueles para avaliar funções executivas, muitos processos podem ser avaliados como atenção, controle inibitório, memória, assim o desempenho do participante na execução do teste pode ser analisado sob diferentes aspectos, como tempo de resposta, tipo de estímulo e número de repetições. A TestPlatform inclui uma bateria de testes de funções executivas, padronizando protocolos de testes e tornando diversos paradigmas acessíveis, proporcionando flexibilidade no uso de diferentes experimentos e precisão nos resultados a serem avaliados. O software foi codificado em C\#, uma linguagem de programação orientada a objetos, e o desenvolvimento do projeto foi conduzido de acordo com a metodologia ágil. O software vem sendo entregue conforme as demandas da pesquisa. Assim, o lançamento inicial consistiu no teste de Stroop na versão original e Victoria, seguido de diversas variações sobre a categoria e a apresentação dos estímulos. Da mesma forma, prosseguiu o teste de tempo de reação, o módulo de teste de escolha e preferência e o seguinte referente ao teste de reconhecimento espacial. A maioria já produziu dados como resultado de projetos de pesquisa na área de Neurociência e Comportamento. Essa plataforma foi estabelecida como uma aplicação desktop de código aberto, largamente utilizada em pesquisas no Laboratório de Neurociência e Comportamento do Instituto de Ciências Biológicas da Universidade de Brasília e distribuída de forma livre em um repositório público.
\end{abstract}

\section{PALAVRAS-CHAVE}

Práticas Ágeis, GitHub, Avaliação Neuropsicológica

\section{INTRODUÇÃO}

Os testes neuropsicológicos são tarefas elaboradas para investigar processos cognitivos, como atenção, memória, linguagem, associados a avaliação de uma estrutura cerebral específica, estabelecendo-se como instrumentos úteis na pesquisa e na neuropsicologia clínica (Seidman, 1998, Zucchella et al., 2018). Escalas comportamentais, categorização, abstração, testes de fluência, testes neuropsicológicos observacionais e testes de raciocínio e aprendizado são frequentemente usados como ferramentas para apoiar o estudo de processos cognitivos frequentemente adotados em ensaios clínicos e procedimentos de pesquisa sobre comportamento, emoções e personalidade que relacionam esses aspectos ao funcionamento cérebro (Harvey, 2012, Ritsner et al., 2006). Os testes neuropsicológicos computadorizados permitem o uso de um paradigma de teste com variações de protocolos, padronizando as condições de apresentação de estímulos e a sua replicação em diversos sujeitos (Duchesne e Mattos, 1997, Charchat et al., 2001). Além disso, permitem uma aplicação com maior precisão no tempo de exposição dos estímulos e intervalos de apresentação, auxiliam no registro das respostas fornecendo para pesquisadores dados para análises quantitativas e qualitativas e possibilitam maior rigor no controle das condiçc̃es de avaliação, provendo maior confiabilidade psicométrica (Ritsner et al., 2006, Capovilla, 2006, Schatz e Browndyke, 2002). 
O projeto e o desenvolvimento da TestPlatform começaram em 2015 no contexto do Programa de Iniciação em Desenvolvimento Tecnológico e Inovação. A motivação inicial para este projeto foi a limitação dos sistemas acessíveis no Laboratório de Neurociência e Comportamento da Universidade de Brasília (UnB). Os pesquisadores eram submetidos a projetar seus estudos com base nas características dos sistemas coexistentes para realização de experimentos com envolvimento de avaliação neuropsicológica. O principal objetivo da TestPlatform é automação da bateria de testes neuropsicológicos aplicados na avaliação de funções executivas, deixando-os flexíveis e parametrizáveis de acordo com as intenções dos experimentos.

As funções executivas (FE) envolvem um conjunto de habilidades cognitivas importantes para a vida cotidiana das pessoas, dependentes da integridade do córtex pré-frontal, a parte anterior do cérebro (Otero e Barker, 2014, DeSouza et al., 2012). As FE compreendem processos de tomada de decisão, motivação, planejamento mental, comportamento na execução de um plano estabelecido e julgamento sobre esse desempenho. O desempenho das FE pode ser verificado a partir de diferentes domínios cognitivos guiados pela atenção, como memória de trabalho, controle inibitório e flexibilidade cognitiva, o que exige testes que se referem ao controle cognitivo de como detectar uma mudança de expectativa, interromper uma resposta, alternar uma resposta, inibir respostas competitivas, detectar conflitos, regular respostas via alerta ou alocação de esforço e manter um objetivo em um período de tempo (Diamond, 2013).

A bateria de testes neuropsicológicos de avaliação de funções executivas é baseada principalmente em paradigmas validados em sua versão clássica. Na plataforma, a bateria está preparada para possibilitar ilimitadas variações de protocolos e adaptações do modelo de referência. Os testes primários contemplados em projeto na plataforma e concebidos de acordo com os requisitos determinados no objetivo do estudo são: o teste de Stroop, baseado no teste clássico de palavras coloridas (ST), para avaliar o controle inibitório, a flexibilidade cognitiva e a memória de trabalho; o teste de tempo de reação (TR), para avaliar o controle inibitório e a flexibilidade cognitiva principalmente; testes de escolha de acordo com o modelo com atraso (DMTS) ou diferente de modelo (DNMTS), para avaliar a memória de trabalho e a flexibilidade cognitiva principalmente; e tarefa de reconhecimento espacial com atraso (TREA), para avaliar memória operacional visuo-espacial.

\section{MATERIAIS E MÉTODOS}

O processo de desenvolvimento de software adotado é baseado no Manifesto Ágil que tem uma dinâmica iterativa e incremental. Os artefatos são gerados regularmente, agregando valor ao produto final e às partes interessadas. Um pesquisador sênior de neurociência desempenha o papel de proprietário do produto (PO), além de fornecer e explicar os requisitos para a equipe de projeto e desenvolvimento, composta por estudantes de engenharia de software. Os pesquisadores de laboratório são os principais demandantes e os principais usuários finais.

A gestão do processo vem sendo cumprida através da ferramenta de projeto GitHub, que registra o fluxo de trabalho dos recursos e tarefas do sistema, ver Figura 1. Os requisitos de software são listados em recursos e tarefas. O método Kanban é utilizado para o gerenciamento de tarefas de desenvolvimento, o que auxilia na documentação dos requisitos (Mariotti, 2012). A priorização de requisitos, a proposta e aprovação de mudanças no projeto são realizadas em reuniões regulares, permitindo a adaptação do sistema às necessidades observadas por diversas partes interessadas, seja cliente, desenvolvedor ou usuário do sistema.

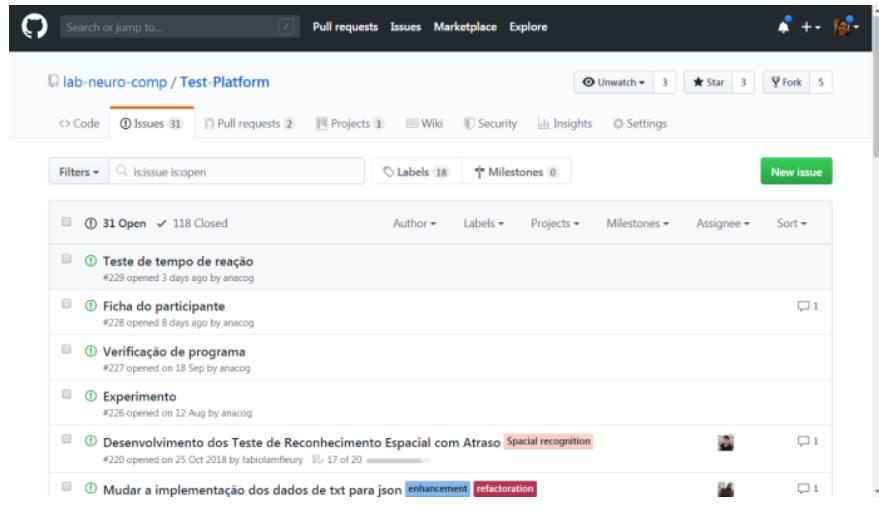

Figura 1. Exemplo de uso do GitHub como ferramenta de gestão no lançamento de pendências 
A plataforma foi estabelecida baseada em diretrizes de projeto que devem ser cumpridos durante todo o desenvolvimento. A arquitetura da tecnologia deve ser flexível e portátil para atender as necessidades da área aplicada e perfil dos usuários, garantindo um arquivo executável capaz de ser executado a partir de qualquer periférico gravável sem instalações extras. O cerne da plataforma, que é a execução dos testes, deve estar livre de conexão à Internet, em favor da qualidade dos dados fornecidos e da portabilidade. O software deve estar disponível de acordo com as entregas relacionadas ao usuário, quantas vezes eles solicitarem, não apenas a partir de um módulo completo. A preocupação com a portabilidade da plataforma é que as avaliações possam ser feitas em uma ampla diversidade de ambientes, como escolas, clínicas, hospitais e laboratórios. Para atender os requisitos fundamentais da arquitetura e projeto, esta aplicação é codificada em C\# usando o Visual Studio, respeitando a programação orientada a objetos. O projeto original da TestPlatform mantinha o foco em aspectos de estímulos, coleta de respostas e estruturas de dados, para que pudesse ser implementado em uma arquitetura viável.

Cada um dos testes mencionados como núcleo da bateria apresenta algumas particularidades, em consideração aos respectivos paradigmas de estudo. Para implementação na plataforma, eles foram ordenados a partir dos que possuíam características mais simples em sua versão básica. O ST insere um elemento de conflito entre os estímulos da tentativa que são apresentados na tela e o indivíduo deve inibir uma resposta em favor de outra de acordo com a instrução, nesse caso, espera-se uma resposta verbal. O TR apresenta um elemento visual em posição aleatória na tela, com um intervalo pré-definido que pode ser fixo ou variável, e solicita-se ao sujeito que reaja pressionando uma tecla. O DMTS/DNMTS, em cada tentativa, apresenta um elemento como modelo e, após um tempo que é denominado atraso, reapresenta o modelo e outro estímulo distrator, o indivíduo deve escolher na tela o elemento igual ao modelo, no caso do DMTS, ou o diferente do modelo, para o DNMTS. Finalmente, o TREA dispõe os elementos em diferentes posições na tela gradativamente em uma tentativa e o indivíduo deve identificar a última posição ocupada.

A modelagem da estrutura conceitual da plataforma partiu de características comuns aos testes que formaram a sustentação de classes para todos os paradigmas a serem desenvolvidos. O protocolo delineado com as definições do estudo é denominado programa, o qual mantém a configuração planejada. Os testes têm a execução baseada na apresentação de estímulos ao qual deve ser controlada a precisão no tempo de exposição, de intervalos e o instante exato de exibição, da mesma forma a categoria e a ordem do estímulo escolhido. Toda execução do teste deve ter um registro de saída com todos os dados definidos no programa do teste, assim como, para os testes que exigem resposta via teclado ou tela, o registro preciso do tempo e do tipo de resposta. As saídas são geradas em planilhas para serem manipuladas pelo usuário. O programa pode ser parametrizado em termos de tamanho, tipo e formato dos estímulos, ordem de apresentação dos estímulos, tempo de exposição e intervalo entre as apresentações dos estímulos, detecção de respostas e tipo de resposta - correção, erro e omissão, além das específicas de cada teste. Além disso, sons de aviso para respostas, exibição ou não do ponto de fixação personalizada em cores e formatos, captura de áudio para análises posteriores das respostas verbais e, um recurso específico para integração com equipamentos de registro de sinais biológicos, que é um marcador no sinal digital referente ao instante da apresentação do estímulo. A plataforma permite a organização de um experimento, combinando diferentes testes ou estágios para executar em uma sessão, configurando o intervalo entre eles. Muitas possibilidades de fornecer maior controle sobre o protocolo administrado, bem como a possibilidade de usar variações do protocolo, mantendo o controle e a qualidade do teste em diferentes contextos.

O projeto é mantido em um repositório do GitHub do Laboratório de Neurociência e Comportamento distribuído em termos livre e aberto, junto com o fluxo de gestão de projeto. Neste repositório está definido o licenciamento e as possibilidades de colaboração, assim como algumas informações de uso da plataforma.

\section{RESULTADOS}

Uma versão inicial limitada da TestPlatform foi feita no início de 2016, trazendo o ST na forma original (Stroop, 1935) e Victoria (Regard, 1981). Essas versões induzem um conflito de resposta porque apresentam palavras coloridas escritas com uma cor diferente, por exemplo a palavra verde escrita em vermelho, na qual o participante é instruído a falar em voz alta a cor da tinta, por exemplo, vermelho, não a palavra escrita. Este teste avalia o comportamento diante de um conflito semântico. Para implementação, foram utilizadas listas de palavras e listas de cores, que poderiam ser combinadas aleatoriamente, gerando palavras escritas com a cor 
diferente da tinta, ou combinadas de forma programada, seguindo a ordem das listas, o modo de execução é definido no programa. Esse primeiro uso do software ocorreu em um estudo de treinamento cognitivo em idosos (Varela et al., 2016). A evolução desse teste inclui legenda nos estímulos, mesmo com palavras como implementado por West e Alain (2000). Assim, mantendo o paradigma inicial, outros pesquisadores propuseram variações do teste usando palavras diferentes das cores (Canabarro et al., 2017), imagens com legendas que apresentavam conflitos emocionais (Sarmiento et al., 2016), originando a versão do Treface, e a imagem com áudio (Garcia et al., 2016), originando a versão do Trefi.

Observando essas diversas possibilidades que podem ser trabalhadas e o contexto das pesquisas realizadas no Laboratório de Neurociência e Comportamento da Universidade de Brasília (UnB), a implementação do Stroop evoluiu considerando variações do tipo de estímulo, bem como a combinação de diferentes programas em um protocolo. O teste possui seis tipos de arranjos de estímulos: (1) palavra, versão clássica e variações de palavras e cores; (2) imagem, conflito tratado no conteúdo da imagem ou em conjunto com legendas; (3) imagem e palavra, apresentação pareada de dois estímulos intercalados por um atraso; (4) palavra e imagem, ainda mais cedo, revertendo a sequência dos estímulos na tentativa; (5) palavra com áudio, apresentação simultânea de palavra e áudio; e (6) imagem com áudio, apresentação simultânea de imagem e áudio. A maioria deles foi criada para apoiar experimentos de pesquisa de projetos de mestrado e doutorado. Na Figura 2, existem dois exemplos de teste stroop usando imagens e podem gravar a resposta de áudio dos sujeitos.

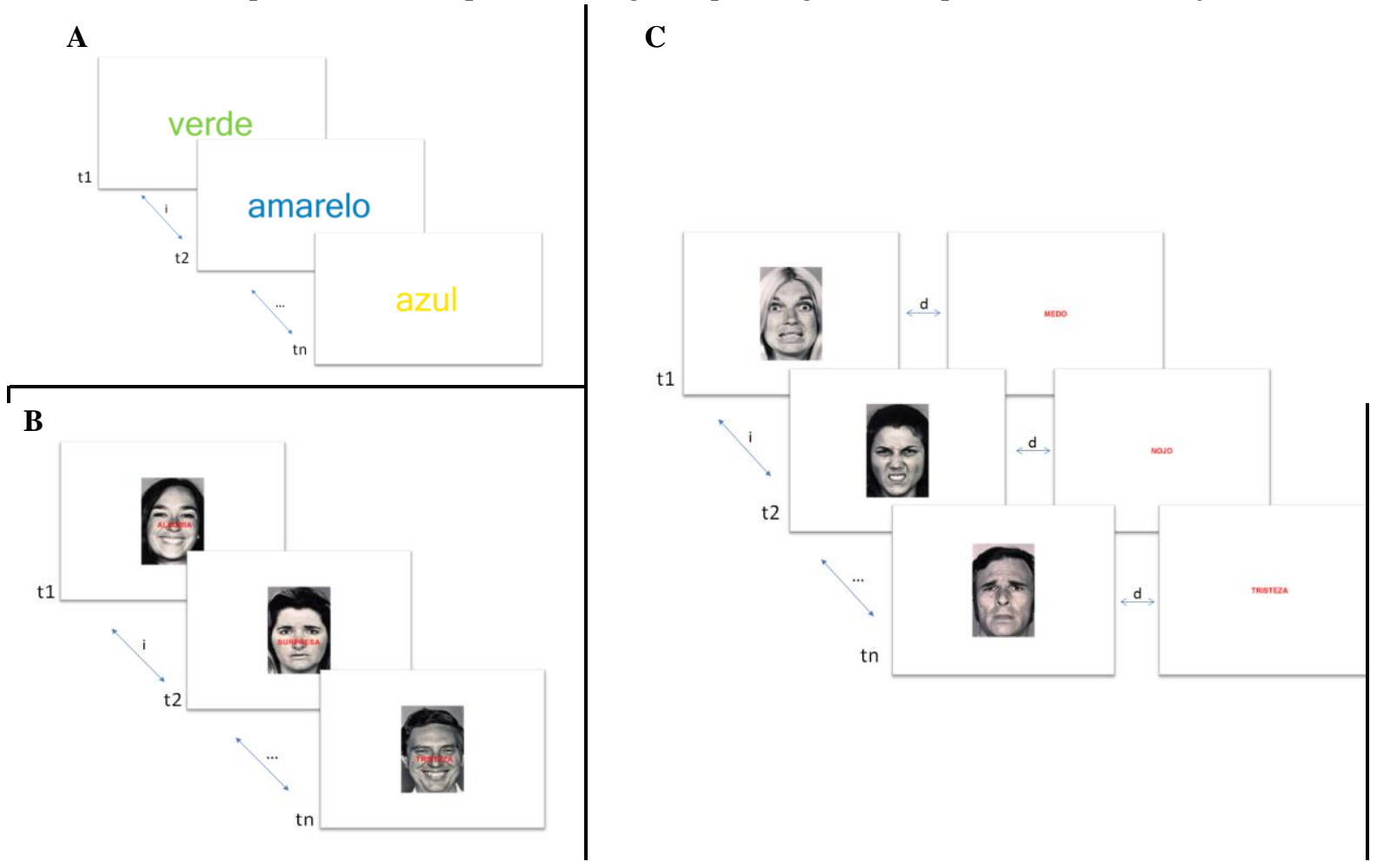

Figura 2. Exemplos de execução do teste Stroop $(\mathrm{t}=$ tentativa; $i=$ intervalo; $d=$ atraso): A. Teste de clássico das palavras coloridas, tipo palavra; B. Treface, tipo imagem com legenda central; C. Treface, tipo imagem com palavra (intercalados com atraso)

O próximo teste desenvolvido foi o TR. O paradigma do tempo de reação foi proposto por Hyman (Hyman, 1953) e avalia a relação entre resposta motora impulsiva e manipulação do tempo. Neste teste, o indivíduo é instruído a pressionar a "barra de espaço" assim que visualizar um estímulo específico na tela do computador que aparecerá em diferentes posições aleatoriamente. O tempo entre a apresentação do estímulo e a detecção da resposta pelo pressionamento da barra será considerado como tempo de reação. A evolução do teste considera uma diversidade de estímulos, além das formas geométricas, como palavras, imagens e áudio com palavra ou imagem, veja a Figura 3, assim como foi incluído o paradigma de tempo de reação com sinal de parada (Logan, 1994), em que o indivíduo é instruído a reagir apenas quando o áudio, o sinal, não for exibido. 


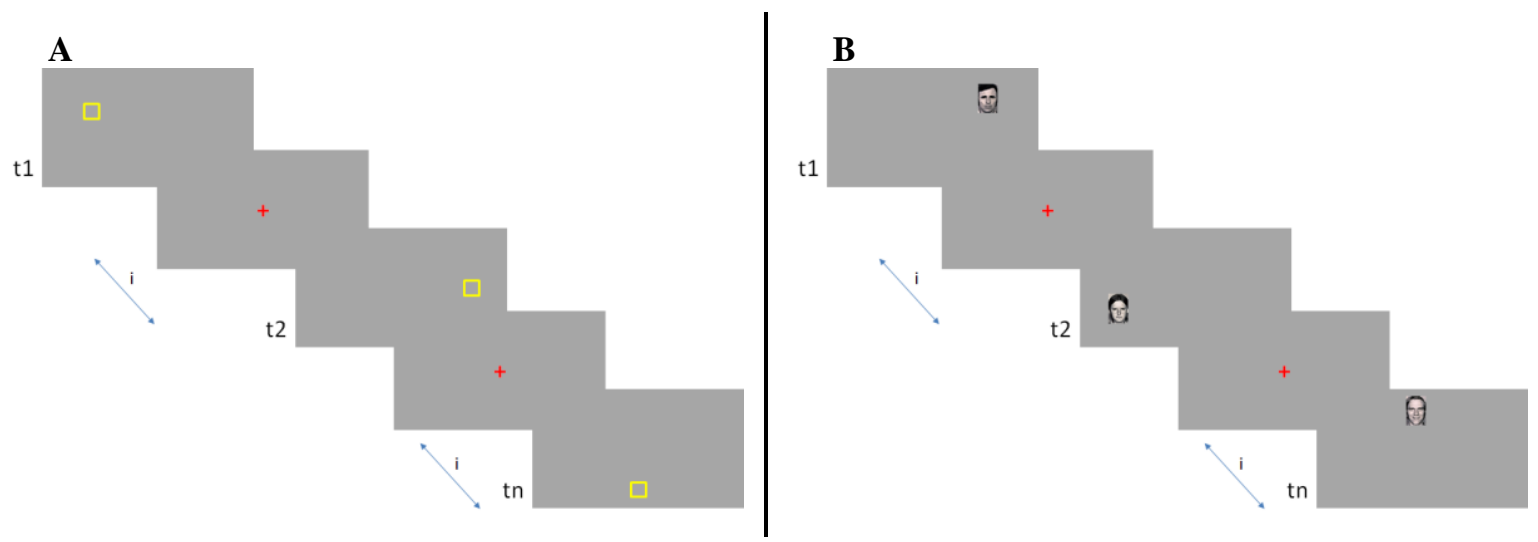

Figura 3. Exemplos de execução do teste de tempo de reação $(\mathrm{t}=$ teste; $\mathrm{i}=$ intervalo): A. forma geométrica de estímulo; B. estímulo de imagem

O TR foi utilizado em um projeto para avaliar o desempenho de jovens estudantes sob efeito de uma neuromodulação com Estimulação Transcraniana por Corrente Contínua (ETCC), utilizando a versão com imagens emocionais (Bernardes et al., 2018).

O seguinte teste a ser concebido na plataforma foi o DMTS/DNMTS baseado no paradigma de escolha de acordo com (ou diferente de) o modelo com atraso (Wright et al., 1986). O procedimento original consiste na apresentação de um estímulo visual na tela, o modelo, seguido de um atraso com uma tela limpa e finalizada pela apresentação de dois estímulos: o modelo e um novo. É avaliada a capacidade de reconhecer o modelo previamente apresentado, obedecendo a regra para a resposta com a escolha ou não do elemento na tela. Na plataforma, esse teste é exposto como teste de escolha, com a opção de execução com ou sem atraso para seleção da resposta. Esta versão sem atraso permite o teste de escolha de acordo com alguns critérios, como preferência, recordação e associação. Além disso, o número de estímulos para correspondência pode ser estendido além do par de estímulos, ver Figura 4.
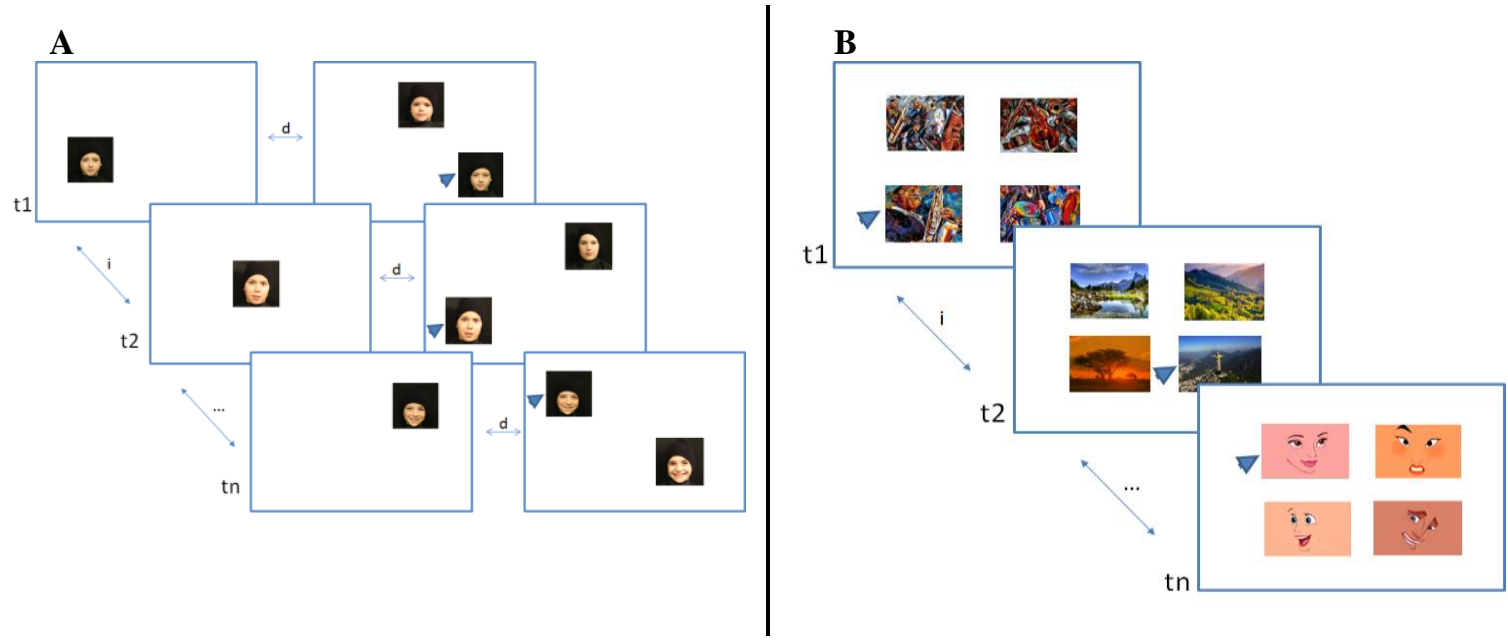

Figura 4. Exemplos de execução do teste de escolha ( $\mathrm{t}=$ tentativa; $\mathrm{i}=$ intervalo; $\mathrm{d}=$ atraso) com indicação da possível escolha: A. escolha de acordo com o modelo com atraso, DMTS; B. teste de escolha com 4 elementos sem atraso

O último bloco de testes previsto no projeto trata da tarefa de reconhecimento espacial com atraso, TREA, baseada no paradigma de Lacreuse e cols. (2005). A tarefa consiste na apresentação de estímulos visuais em uma série de posições aleatórias na tela, o participante deve indicar a última posição preenchida. Na plataforma, além da variedade na categoria do estímulo já praticada nos testes anteriores, foi incluída uma versão sequenciada na qual os participantes devem selecionar todos os elementos na ordem em que foram apresentados na tentativa. Uma tentativa possui tantos passos quanto o número de elementos escolhidos para aparecer; quando ocorre uma seleção incorreta, a tentativa é interrompida e é iniciada a próxima. A Figura 5 representa um teste completo de duas versões do TREA. 

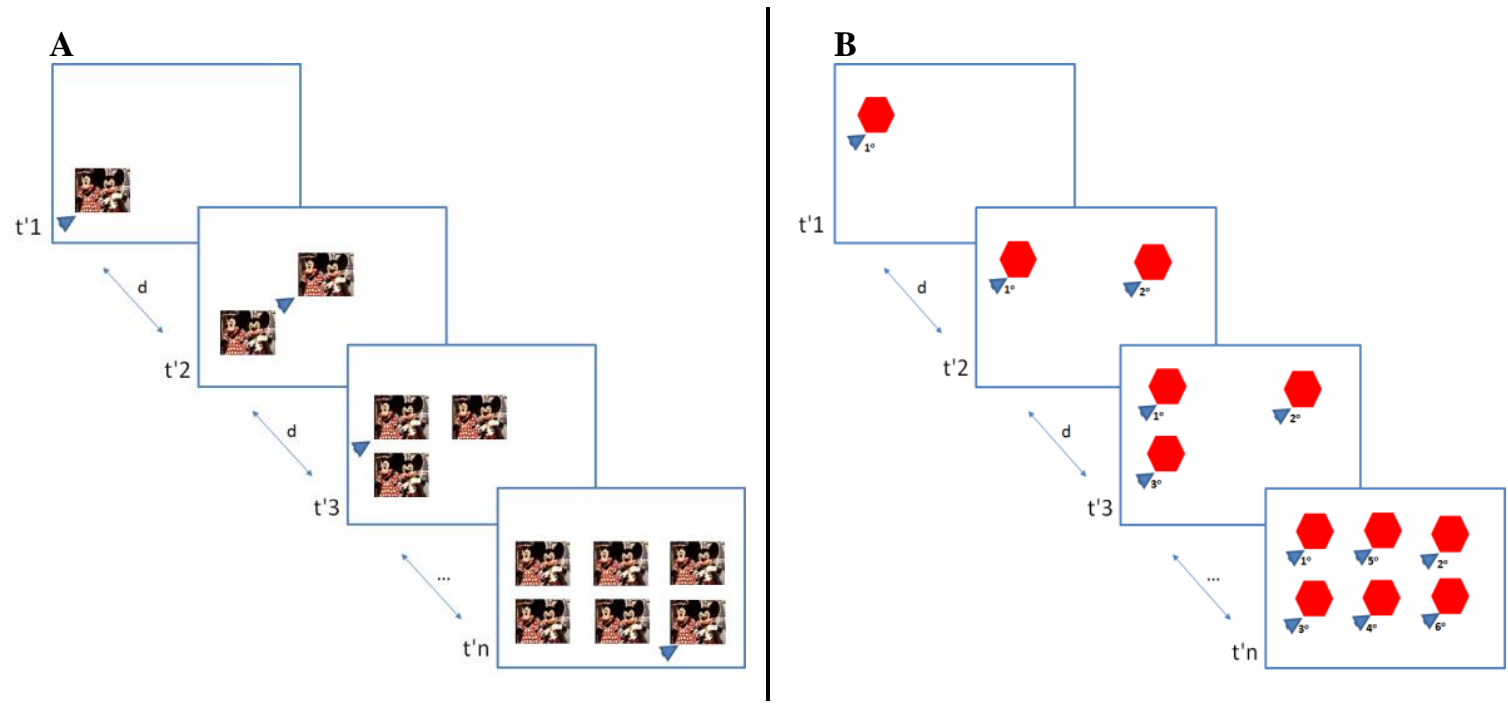

Figura 5. Exemplos de execução do TREA ( $\mathrm{t}$ '= passo dentro do teste; $\mathrm{i}=$ intervalo; $\mathrm{d}=$ atraso) com indicação da seleção: A. a tarefa de reconhecimento espacial com o mesmo estímulo visual; B. a tarefa de reconhecimento es pacial para seleção sequenciada

A maioria dos projetos do Laboratório combina paradigmas diferentes para compor um protocolo de pesquisa. Para dar suporte a esses problemas, foi fornecida a funcionalidade de criar um experimento, que permite a execução de diferentes programas de teste em sequência, ou em ordem aleatória, com instruções específicas e intervalos estabelecidos entre eles. A plataforma registra em formato de tabela todos os dados gerados pela execução dos testes, com detalhes de cada tentativa, bem como do experimento, e é possível manipulá-los em planilhas e pacotes estatísticos compatíveis.

A plataforma também permite coletar informações clínicas e demográficas dos participantes para triagem e análise futuras de seu estado no momento do teste, prática comum em protocolos de pesquisa. Todos os protocolos criados na plataforma podem ser importados e exportados para serem usados em outros computadores, formando, também, um catálogo de estudos.

A TestPlatform permanece em desenvolvimento contínuo, de acordo com as sugestões e necessidades do laboratório e dos usuários. A plataforma é distribuída nas versões em inglês (en-US) e em português (pt-BR) e é aberto a outros idiomas, adicionando texto de tradução no item Globalização no repositório do GitHub.

\section{CONCLUSÃO}

A TestPlatform é uma plataforma de testes computadorizados para avaliação cognitiva na área de neurociências e comportamento. Tem como fundamento fornecer uma ampla bateria de testes para avaliar funções executivas em pesquisas do Laboratório de Neurociências e Comportamento do Instituto de Ciências Biológicas da UnB, permitindo diversas adaptações dentro de cada paradigma. Como a aplicação já vem sendo utilizada por pesquisadores do laboratório, tem sido possível realizar um desenvolvimento orientado às necessidades dos usuários e validação contínua, mantendo a equipe integrada e multidisciplinar, com a participação dos pesquisadores do laboratório, como fornecedores de requisitos e necessidades de melhoria. As atualizações têm sido predominantes em termos de novas funcionalidades para atender soluções que estão sendo avaliadas ou implementar ideias para futuros estudos, posto que todo programa de teste deve ser parametrizável em todos os detalhes requeridos. Desta forma, a admissão da plataforma permitiu uma versatilidade no desenho experimental, uma vez que o pesquisador não se limita mais aos recursos da ferramenta disponível, o que viabiliza a diversificação dos estudos e mantém um grande inventário de opções de arranjos nos teste. A TestPlatform pretende favorecer pesquisas e avaliações clínicas executando testes, simplificando-os e fornecendo dados confiáveis e precisos, permitindo que os pesquisadores direcionem sua atenção para análise dos resultados. 


\section{REFERÊNCIAS}

Bernardes, T. S., Tavares, M. C. H., Garcia, A., SILVA, C. R., Fleury, F. M., Brasil Neto, J. P., 2018. Estimulação Transcraniana por Corrente Contínua (ETCC) Pré-frontal e Tempo de Reação: Um Estudo Piloto. X Simpósio Internacional de Neuromodulação, São Paulo, Brasil.

Canabarro, S. L. S., Garcia, A., Satler, C., Tavares, M. C. H., 2017. Interaction between Neural and Cardiac Systems during the Execution of the Stroop Task by Young Adults: Electroencephalographic Activity and Heart Rate Variability. AIMS Neuroscience, 4(1): 28-51. doi: 10.3934/Neuroscience.2017.1.28.

Capovilla, A., 2006. Desenvolvimento e validação de instrumentos neuropsicológicos para avaliar funções executivas. Avaliação Psicológica, 5(2), 239-241.

Charchat, H., Nitrini, R., Caramelli, P., Sameshima, K., 2001. Investigação de Marcadores Clínicos dos Estágios Iniciais da Doença de Alzheimer com Testes Neuropsicológicos Computadorizados. Psicologia: Reflexão e Crítica, 14(2), 305-316.

DeSouza, J. F.X., Shima. O., Pynn, L. K., 2012. Correlating behavioral responses to fMRI signals from human prefrontal cortex: Examining cognitive processes using task analysis. Journal of visualized experiments: JoVE 64. Jun 20;(64). pii: 3237. doi: 10.3791/3237.

Diamond, A., 2013. Executive functions. Annual Review of Psychology, 64, 135-168.

Duchesne, M., Mattos, P., 1997. Normatização de um teste computadorizado de atenção visual. Arquivos de Neuropsiquiatria, 55(1), 62-9.

Garcia, A., Medeiros, W., Garrido, L. M. M., Tomaz, C. A. B., Tavares, M. C. H., 2016. Uma proposta para Avaliação da Memória Emocional com um Teste de Reconhecimento Espacial com Atraso em Crianças com Transtorno de Déficit de Atenção/Hiperatividade. XV Congresso Brasileiro da SBNp, Brasília, Brasil.

Harvey P. D. (2012) Clinical applications of neuropsychological assessment. Dialogues in Clinical Neuroscience, 14, 91-99.

Hyman, R. (1953)Stimulus information as a determinant of reaction time. Journal of experimental psychology, 45(3), 188.

Lacreuse A, Kim C. B., Rosene D. L., Killiany R. J., Moss M. B., Moore T. L., Chennareddi L., Herndon J. G. (2005) Sex, age and training modulate spatial memory in the rhesus monkey (Macaca mulatta). Behavioral Neuroscience, 119(1), $118-26$.

Logan, G.D. (1994). On the ability to inhibit thought and action: A users' guide to the stop signal paradigm. In D. Dagenbach \& T.H. Carr (Eds), Inhibitory processes in attention, memory, and language. Academic Press (pp. 189-239). San Diego, USA.

Mariotti, F. S., 2012. Kanban: o ágil adaptativo. Engenharia de Software Magazine, 45(4),6-10.

Otero, T., Barker, L., 2014. The Frontal Lobes and Executive Functioning. In S. Goldstein \& J. A. Naglieri (Eds.). Handbook of executive functioning. Springer (pp. 29-44). New York, USA.

Regard M., 1981. Cognitive rigidity and flexibility: A neuropsychological study. Unpublished doctoral dissertation, University of Victoria, Victoria, Canadá.

Ritsner, M. S., Blumenkrantz, H., Dubinsky, T., \& Dwolatzky, T., 2006. The detection of neurocognitive decline in schizophrenia using the Mindstreams. Schizophrenia Research, 82(1), 39-49.

Sarmiento, E. L. P., Satler, C., Tomaz, C., 2016. Avaliação da memória operacional no Teste de Stroop de Expressões Faciais com conflito emocional (TREFACE). XV Congresso Brasileiro da SBNp, Brasília, Brasil.

Schatz, P., Browndyke, J., 2002. Applications of computer-based neuropsychological assessment. Journal of Head Trauma Rehabilitation, 17(5), 395-410.

Seidman, L. J., 1998. Neuropsychological testing. Harvard Mental Health Letter, 14 (11), 4-6.

Stroop, J. R., 1935. Studies of interference in serial verbal reactions. Journal of Experimental Psychology, 18(6), 643.

Varela, M., Faria, E., Rabelo, G., Mendes, K., Levino, L., Garcia, A., Tavares, M. C. H., Satler, C., 2016. Avaliação de diferentes processos inibitórios em uma amostra de idosos saudáveis. . XV Congresso Brasileiro da SBNp, Brasília, Brasil

West, R., Alain, C., 2000. Effects of task context and fluctuations of attention on neural activity supporting performance of the Stroop task. Brain Research, 873(1), 102-111.

Wright, A. A., Urcuioli, P., \& Sands, S. F.,1986. Proactive interference in animal memory. In D. F. Kendrick, M. E. Rilling, \& M. R. Denny (Eds.), Theories of animal memory. Erlbaum (pp. 101-125). Hillsdale, USA.

Zucchella, C., Federico, A., Martini, A., Tinazzi, M., Bartolo, M., Tamburin, S., 2018. Neuropsychological testing. Practical Neurology, 18:227-237. 